\title{
Challenges encountered during the management of pediatric facial soft tissue injury from a mobile battery blast: A case report
}

\author{
Rikta Pande ${ }^{1}$, Bandana Koirala ${ }^{1}$, Mehul Jaisani ${ }^{1}$, and Chandrakant Pasvan ${ }^{1}$ \\ ${ }^{1} \mathrm{BP}$ Koirala Institute of Health Sciences
}

May 11, 2021

\begin{abstract}
The present case reports facial soft tissue injury involving the lateral aesthetic unit of the cheek on a 10-year-old boy from the disposed mobile battery blast on a roadside campfire. This case highlights one of the challenges encountered from injury to parotid duct and its conservative management.
\end{abstract}

Challenges encountered during the management of pediatric facial soft tissue injury from a mobile battery blast: A case report

Rikta Pande, ${ }^{1}$ Bandana Koirala, ${ }^{2}$ Mehul Jaisani, ${ }^{3}$ Chandrakant Pasvan ${ }^{4}$

${ }^{1 \& 2}$ Department of Pedodntics and Preventive Dentistry, B.P.K.I.H.S, Dharan, Nepal,

${ }^{3 \& 4}$ Department of Oral and Maxillofacial Surgery, B.P.K.I.H.S, Dharan, Nepal,

Correspondence: Dr. Rikta Pande,

Department of Pedodontics and Preventive Dentistry,

B.P.K.I.H.S, Dharan, Nepal,

E-mail address: riktapande@gmail.com

Phone Number: +9779841744510

Abstract: Soft tissue injuries account for the majority of the pediatric maxillofacial injuries. Although rarely life-threatening, the treatment of these injuries can sometimes be complicated and may have a significant impact on the patient's facial function and aesthetics. There are multiple etiologies for trauma where sociodemographic, cultural and environmental factors play an important role in its occurrence. The present case reports facial soft tissue injury inflicted upon a 10-year-old boy from the disposed mobile battery blast on a roadside campfire. The injury involved the lateral aesthetic unit of the cheek, where the presence of the parotid gland and its duct can complicate the surgical management if careful and early assessment of the wound and use of appropriate surgical techniques are not followed. The present case reports one of the challenges encountered from injury to parotid duct and its management.

Key words : Lateral aesthetic unit of cheek; mobile battery blast; parotid gland; pediatric maxillofacial injuries

Key Clinical Message : In case of a deep subcutaneous wound of the cheek region, due consideration on parotid gland injury is recommended. At times, a simple conservative approach turns out to be effective in managing complications of this nature.

INTRODUCTION 
Facial trauma in children is a relatively common occurrence. In the present case, facial injury resulted from the disposed mobile battery blast on a roadside campfire and involved the lateral aesthetic unit of cheek, which represents region between the buccal unit and auricle. This unit is covered with skin that adheres more to the underlying fascia, hence can complicate the re-construction. ${ }^{1}$ Concurrently, deep to the dermal layer there are delicate anatomical structures such as parotid gland and facial nerves, which can complicate the surgical management if early assessment of the wound and use of appropriate surgical techniques are not followed.

\section{CASE REPORT}

A 12-year-old male child reported to the Pediatric Emergency with a history of mobile battery blast injury on the right side of the face six hours after the incident. There was a negative history of loss of consciousness and nausea/vomiting following the incident. Past medical and drug history were non-significant.

\section{On examination:}

Extraorally on right lateral unit of the cheek, there was a circular injury of approximately $3 \mathrm{~cm}$ in diameter which was communicating intraorally to the oral cavity. There was herniation of the buccal pad of fat, blood clots, and superficial burn around the wound (Fig 1). Intraorally, on the right buccal mucosa laceration of around $1 \mathrm{~cm}$ along with the herniated buccal pad of fat was present. The parotid gland appeared to be injured however, facial and trigeminal nerve functions were intact. No injuries were present on other body parts. Based upon the findings, a clinical diagnosis of soft tissue injury ${ }^{2}$ was made.

\section{Management:}

The patient was kept under antibiotic and analgesic therapy with injection ceftriaxone (750mg/BD) and injection ketorolac $(20 \mathrm{mg} / \mathrm{TDS})$. The patient also received a booster dose of tetanus toxoid immunoglobulin.

Multidisciplinary team of Oral and Maxillofacial surgery and Department of Pedodontics worked under general anesthesia for primary wound closure in emergency operation theater. Initially cleansing and rinsing of the wound was done using povidone-iodine and normal saline solution, followed by marginal necrosectomy and debridement. Wound closure was done at multiple layers. Suturing of the oral mucosa, parotid gland, parotid capsule, and sub-dermal layer was done with 4-0 polyglactin suture while 5-0 prolene suture was used on the superficial skin layer.

Post-operatively, patient was supported with antibiotics, analgesics, and fluid supplements and dressing was done twice a day for 1 week.

\section{Post-operative Complications:}

On the fourth post-operative day, patient developed salivary leakage from the extraoral margins of the sutured wound (Fig 2). Initially, the leakage was attempted to be managed using pressure dressing. Also re-suturing of the margins were performed under local anesthesia at the drainage site on the seventh postoperative day. Pressure dressing was continued for one week which resulted in stoppage of the leakage along with the recovery of the patient. Finally on the fourteenth post-operative day, sutures were removed and the patient was discharged in a healthy state (Fig 3).

\section{DISCUSSION:}

Facial soft tissue injuries are a common occurrence in the pediatric population. Although rarely lifethreatening, treatment of these injuries can be complex and may have a significant impact on the patient's facial function and aesthetics. ${ }^{3}$

Management principles for soft tissue injuries in children are almost similar as for the adults. In the case, initial wound care was started with copious irrigation and debridement of the devitalized and necrotic tissues. Wound repair was done in multiple layers. Absorbable 4/0 or $5 / 0$ vicryl or polydioxanone sutures (PDS) is suitable for muscle and subcutaneous tissue. Whereas, prolene/nylon $6 / 0$ is the choice for skin approximation. ${ }^{4}$ 
The cheeks are by surface area the largest subunit of the face. This size correlates with a high frequency of injury to the cheek and the underlying structures. ${ }^{3}$ Parotid duct injury should be considered in any deep injuries to the cheek area located inferior to a line extending from the tragus to the upper lip. Early detection and repair of the ductal injuries is strongly advised since complications from delayed identifications are challenging. The signs of unrecognized parotid duct injury can be erythema, edema, sialocele, and tenderness after uneventful soft tissue repair. ${ }^{5}$

Post-traumatic salivary fistulas, sialoceles or leakages are initially managed with conservative treatment which includes pressure dressing, needle aspiration, and administration of broad spectrum antibiotics. A series by Lewis and Knottenbelt ${ }^{6}$ concluded that conservative management was adequate for parotid duct injuries. Similarly, Landau and Stewart ${ }^{7}$ concluded that systemic probanthine, intravenous fluids, a nil by mouth regimen, and external pressure would produce resolution of symptoms, even in the presence of a fistula or sialocele. In the current case also, re-suturing at the drainage site followed by pressure dressing for one week brought about the favorable result.

The present case also raised our attention towards the mishaps from the electronic wastes. Hence, preventive measures and education to the individual, parents and society on a whole regarding the etiologies of trauma in children and the proper disposal of E-wastes becomes very crucial.

\section{CONCLUSION:}

Parotid gland injury should always be considered whenever a case of deep subcutaneous injury on cheek region is encountered. Parotid duct was involved in the present case of facial trauma as well and the complication arising from the injured parotid duct in the case was detected and managed successfully using the conservative approach of compression dressing.

\section{Author Contribution}

Rikta Pande: Patient management \& manuscript write-up

Chandrakant Pasvant,Mehul R Jaisani- Primary patient management and patient follow-up.

Bandana Koirala: Manuscript editing and proof reading

Conflict of Interest: None

Acknowledgement: Department of Oral and Maxillofacial Surgery

\section{REFERENCES}

1. Kretlow J, McKnight A, Izaddoost S. Facial Soft Tissue Trauma. Semin Plast Surg. 2010 Nov;24(04):348-56.[PubMed |DOI]

2. Euerle B, Kelly B. Maxillofacial Injuries: Clinical Characteristics and Initial Management. Trauma Reports. 2005 May;6(3):1-2.[Google Scholar]

3. Kretlow J, McKnight A, Izaddoost S. Facial Soft Tissue Trauma. Semin Plast Surg. 2010 Nov;24(04):348-56.[PubMed |DOI]

4. Bhattacharya V. Management of soft tissue wounds of the face. Indian J Plast Surg. 2012 Sep;45(3):436-43. [PubMed|DOI ]

5. Ambro BT, Azola AM, Perry K. Pediatric Soft Tissue Injuries and Management. In: Encyclopedia of Otolaryngology, Head and Neck Surgery. 2013. p. 2106-15. [Springer Link|DOI]

6. Lewis G, Knottenbelt JD. Parotid duct injury: is immediate surgical repair necessary? 1991;22(5):4079. [PubMed |DOI ]

7. Parekh D, Glezerson G, Stewart M, Esser J, Lawson HH. Post-traumatic parotid fistulae and sialoceles. A prospective study of conservative management in 51 cases. Ann Surg. 1989];209(1):105-11. [PubMed |DOI ] 

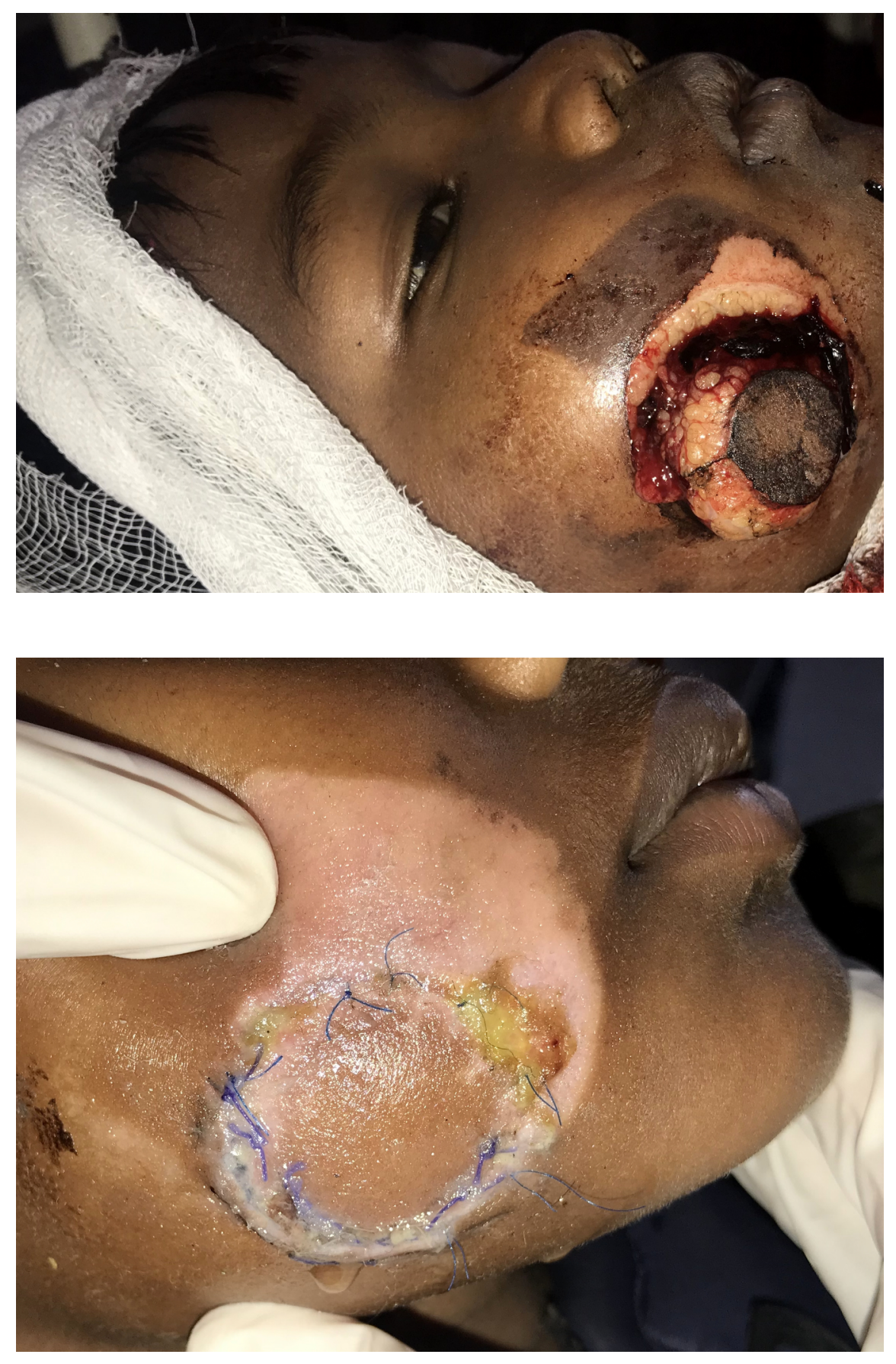

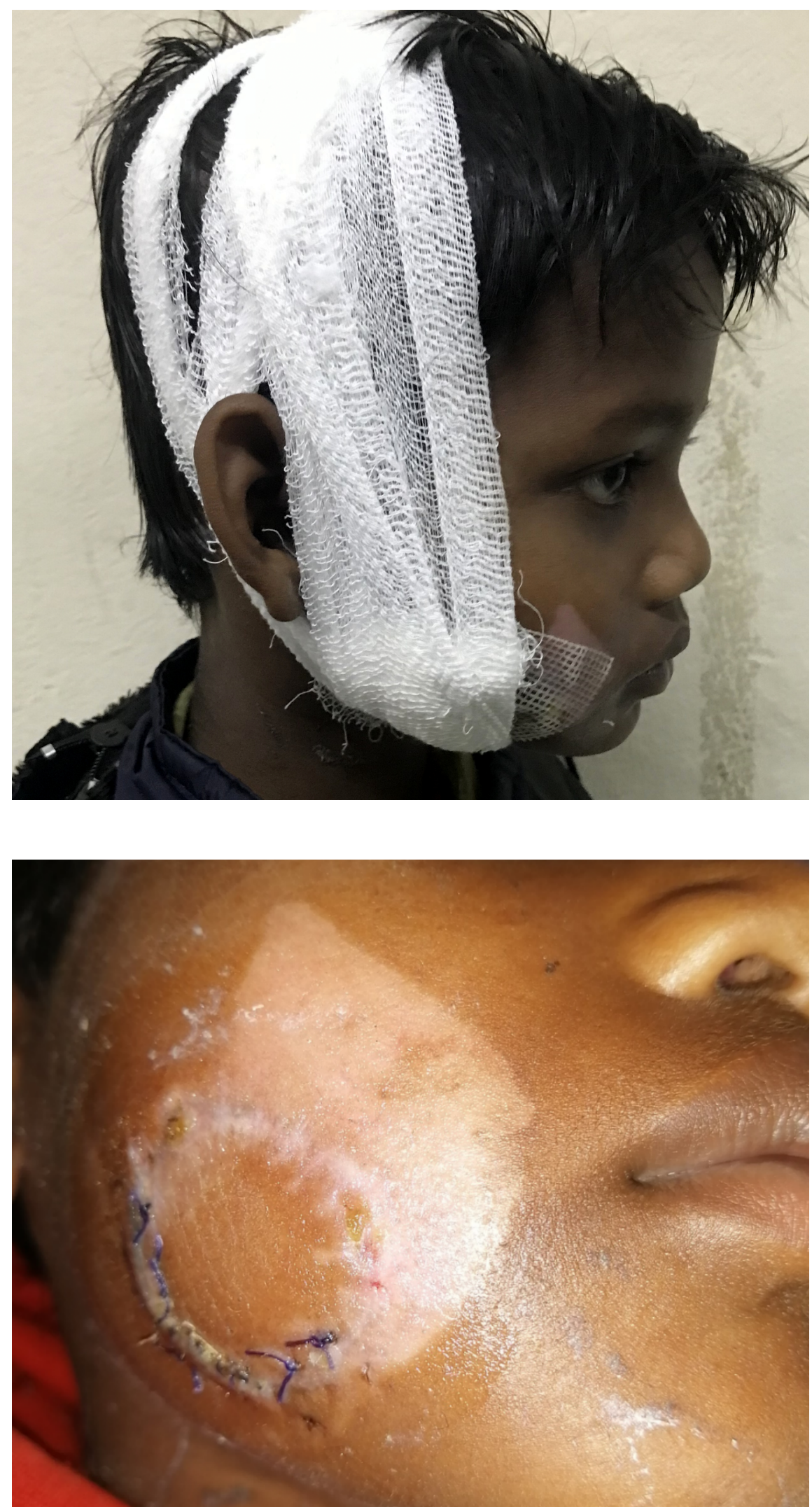